\title{
Une constance à la chinoise : Considérations sur l'art performatif extrême chinois
}

\section{Erik Bordeleau}

\section{(2) OpenEdition \\ 12 Journals}

Édition électronique

URL : http://journals.openedition.org/transtexts/269

DOI : $10.4000 /$ transtexts.269

ISSN : 2105-2549

Éditeur

Gregory B. Lee

Édition imprimée

Date de publication : 1 juin 2009

ISSN : 1771-2084

Référence électronique

Erik Bordeleau, « Une constance à la chinoise : Considérations sur l'art performatif extrême chinois », Transtext(e)s Transcultures 跨文本跨文化 [En ligne], 5 | 2009, document 3, mis en ligne le 03 avril 2010, consulté le 10 décembre 2020. URL : http://journals.openedition.org/transtexts/269 ; DOI : https:// doi.org/10.4000/transtexts.269 


\title{
Transtext(e)s Transcultures 跨文本跨文化
}

Journal of Global Cultural Studies

5 | 2009:

Varia

(Re)Inventing "Realities" in China

\section{Une constance à la chinoise : Considérations sur l'art performatif extrême chinois}

\author{
ERIK BORDELEAU
}

\section{Résumés}

Cet article se propose de rendre compte de l'évolution fulgurante de l'art performatif chinois à partir du début des années 90 jusqu'au début des années 2000, période relativement brève mais qui en constitue sans doute l'apogée. La radicalité et le caractère extrêmement controversé de certaines des performances discutées ici nous amène à nous questionner sur ce qui différencie les performances qui se présentent comme expression d'un malaise politico-existentiel et celles qui agissent comme acting-out d'une logique biopolitique. Notre travail s'articulera autour des questions suivantes : quels sont les enjeux impliqués dans l'expositionperformative d'une conduite humaine? Ou dans une optique plus biopolitique: que signifie exposer/dis-play (de) l'humain? Cette réflexion sur l'art performatif en Chine dans une optique biopolitique et anthropogénétique s'appuiera principalement sur les œuvres de Yang Zhichao, He Yunchang, Zhang Huan, Peng Yu, Sun Yuan et Zhu Yu.

At the turn of the 21st century, Chinese extreme performative art reached unprecedented level of 
radicality and controversy. In this article, I will try to mark a difference between performances which present themselves or can be interpreted as expressions of political and existential malaise, and those that could be thought of as a sort of acting-out of the biopolitical logic. My article will revolve around the following questions: what is at stake in the performative exhibition of human behaviour, as the Chinese translation of the term "performative art" (xingwei yishu) suggest? Or in a more biopolitical perspective: what does it mean to dis-play "some" human? This reflection on Chinese performative art in a biopolitical and anthropogenetical perspective will be based principally on the work of Yang Zhichao, He Yunchang, Zhang Huan, Peng Yu, Sun Yuan and Zhu Yu.

\section{Texte intégral}

Pour civiliser l'esprit, il faut d'abord ensauvager le corps. Mao Zedong, Une étude de l'éducation physique (1917)

Dans cet article, nous nous proposons de rendre compte de l'évolution fulgurante de l'art performatif chinois à partir du début des années 90 jusqu'au début des années 2000, période relativement brève mais qui en constitue sans doute l'apogée. La radicalité et le caractère extrêmement controversé de certaines des transgressions opérées par les performances que nous allons discuter, transgressions qui plus est performées dans un contexte sociopolitique a priori réfractaire à ce genre de pratiques artistiques, en font des objets d'analyse politique presque trop évidents. On peut aisément interpréter l'art performatif en Chine dans l'optique d'une expression directe du malaise politique régnant dans la Chine post-Tiananmen. Une tension nette s'établit alors entre les dispositifs de contrôle social et des performances exutoires enracinées dans le monde de la vie, qui agissent comme une sorte de contre-effectuation émancipatrice de la violence étatique et son pouvoir d'extraction de vie nue. On pourrait dire que la puissance expressive de ces performances et leur potentiel d'émancipation propre résident dans leur capacité à exposer et reproduire intentionnellement l'événement de cette violence, par la mise en danger (en survie) de corps dans lesquels elle s'est inexorablement disséminée. Cependant, vers la fin des années 9o, une nouvelle vague de performances compliquera les choses, ajoutant une dimension proprement biopolitique à la scène performative chinoise. Dans ce travail, nous essaierons d'élucider ce passage, en posant une distinction conceptuelle entre les performances du corps-soi et les performances du corps-chair.

Cette discussion de quelques unes de ces performances extrêmes, dont plusieurs ont été sévèrement condamnées pour leur insupportable cruauté, tient lieu sur fond d'une réflexion plus générale sur la nature de l'acte performatif et son inscription dans le contexte culturel chinois. Au fil de l'écriture, la question du rapport entre performance et politique s'est peu à peu constituée comme passage (particulièrement angoissant) sur la ligne de la forme de l'humain, suivant peut-être l'étonnement profond et maintes fois renouvelé que suscite le fait, à première vue banal, qu'en Chine, l'expression «art performatif » est généralement traduit par 行为艺术 (xingwei yishu), qui signifie quelque 
chose comme « art relatif au comportement », ou " art béhavioral » (précisons qu'ailleurs en Asie de l'Est, on traduit généralement par 行动艺术 (xingdong yishu), littéralement action art). L'idée de comportement renvoie directement à la tradition confucéenne, pour laquelle toute conduite individuelle comporte une dimension rituelle qui exprime une certaine position dans l'ordre social. Gao Minglu a souligné avec beaucoup de clarté les implications sociopolitiques liées à ce choix terminologique :

This traditional concept imparted to the Chinese performance art scene has inherently (or congenitally) a social significance. This social content is always present regardless of whether or not an individual performance artist attempts to express certain social content or not in his/her specific performance work. ${ }^{1}$

En traduisant « performatif » par « comportement » ou plutôt « conduite », les artistes chinois continentaux ont résolument signifié leur intention de confronter les règles régissant la conduite des corps dans l'espace public. Restant au plus près du mot xingwei, on pourrait le traduire par « démarche intentionnée » ou littéralement « démarche pour »; pensons par exemple à 人为renwei, qui souligne la qualité proprement humaine, et par extension, artificielle, d'un effort ou d'une entreprise. Wei implique un « en tant que » qui constitue un plan de représentation propre au comportement ou à la conduite humaine. Rapporté à xing, démarche, il en signifie le pour quoi.

$4 \quad$ En redoublant le plan de représentation éthique sur lequel se constitue l'humain, le xingwei yishu affirme haut et fort la contingence radicale de l'individualité, ce qui vient effectivement troubler une certaine idée de la cohésion ou «harmonie » sociale, spécialement en contexte chinois. Si on ajoute à cela l'immédiateté corporelle disruptive propre à la performance, on comprend aisément pourquoi elle est rapidement devenue un des moyens d'expression privilégiés pour la critique sociale en Chine :

From Traditional Confucian ethics to the Cultural Revolution of the Maoist era to the current era of globalization, the behaviour of the individual has been continually seen as meaningless, albeit from different perspectives; thus the body may have been seen by the Chinese avant-garde as an active site of resistance to this constant obliteration of the self. ${ }^{2}$

Le rejet de l'art performatif par les institutions artistiques chinoises a également conduit à en faire un médium de choix pour les artistes voulant exprimer leur critique sociale. Cette censure a, ironiquement, contribué à donner une plus grande visibilité à cette forme d'art, qui jusqu'à tout récemment, était strictement interdite en Chine.

6 Le remarquable pragmatismede la traduction de « art performatif » par « art comportemental » apparaîtra peut-être réducteur à un lecteur occidental. Si elle permet de penser directement la dimension transgressive de l'art performatif, son va-et-vient subversif entre les sphères privée et publique et sa remise en question radicale des règles de transmission et de production culturelle, on peut par contre craindre qu'en moralisant excessivement 
l'acte performatif, elle fasse perdre de vue son idéalité événementielle - quelque chose comme sa détermination historique propre en tant que pratique artistique hypermoderne. Ne faudrait-il pas dès lors lui préférer l'expression 现场艺术 (xianchang yishu), art du «ici et maintenant » ou live art comme certains l'ont proposé?3

Discutant avec un ami chinois de mes recherches sur ce thème, il me raconta l'histoire d'un lettré du 3 ème siècle après J.-C. dénommé Liu Ling, considéré comme un des « Sept sages de la forêt de bambou ». À une époque de grande turbulence politique, ces lettrés taoïstes ont choisi une vie retirée, en nature, affranchie des rites contraignants et des servitudes de la vie politique qui réglaient dans les moindres détails l'existence des lettrés chinois. Ils incarnent ainsi un idéal de spontanéité et de liberté individuelle qui a été très influent dans le monde des arts chinois, jusqu'à aujourd'hui. On n'a qu'à penser par exemple aux 5 films qui composent la série Sept intellectuels dans une forêt de bambou (2003-07) de Yang Fudong, qui réactualise ce mouvement taoïste dit de la « conversation pure » dans un contexte d'art contemporain.

Liu Ling s'est rendu célèbre pour avoir déclaré : 《我以天地为栋宇, 屋室为裤衣。诸君何 为入我裤中? » (traduction libre : "Le ciel et la terre sont ma maison, et cette chambre est mon pantalon. Messieurs, que faites-vous dans mes pantalons? ») Drapé dans l'univers, l'ivrogne-poète s'exhibe nonchalamment. Difficile de dire exactement ce qui a incité mon ami à me faire part de cette savoureuse anecdote. Est-ce parce qu'elle met en scène une nudité scandaleuse, laquelle en Chine est plus souvent qu'autrement associée à la performance? ${ }^{4} \mathrm{Ou}$ serait-ce parce qu'elle présente un comportement qui à la fois ébranle et performe avec force poésie la situation intermédiaire de l'humain dans la cosmologie chinoise - l'humain comme trait d'union imaginaire entre le ciel et la terre, selon le principe du 天人感应 (tianren ganying)? On serait peut-être tenter de lui répondre avec la suffisance de celui qui s'adresse au non-initié : "Ceci n'est pas une performance. C'est un anachronisme, tout au plus », et de déplorer l'égarement auquel la traduction de «art performatif » par xingwei yishu a pu contribuer. Et, secrètement peut-être, retenir contre cet énergumène exhibitionniste de ne pas savoir aussi bien que nous, médianoïdes hypermodernes, ce que cela signifie, (s)'exposer.

Mais si ce n'était pas, au contraire, la définition même - l'institution esthétique - de l'art performatif qui viendrait elle-même problématisée dans ce rapprochement inusité entre des pratiques hypperréflexives hautement qualifiées et dites "performatives", et la boutade d'un mythique ivrogne-poète? Il y a une forte dose de conscience transcendantale qui couve dans le display performatif, sorte d'option spirituelle pro-analytique contemporaine où l'hyperréflexivité tient lieu d'éveil au corps dont on aurait trop longtemps négligé l'importance. Ce corps, entre-temps devenu le référent royal d'un certain art contemporain et l'objet d'une infinité d'études socioculturelles, ce corps qu'on se représente de manière performative pour d'autant mieux en "prendre conscience » et qui constitue ainsi l'ultime objet de l'hégémonie «consciensuelle » de notre époque, ce 
corps qui, paradoxalement, apparaît ainsi comme l'ultime avatar métaphysique qui n'en finit plus de nous hanter, ce corps trouve dans le contexte chinois une troublante réfraction dans les pratiques de soi d'inspiration bouddhiste et taoïste, ou, simplement, dans la pratique traditionnelle de la calligraphie - précisément parce que le « corps » n’y a jamais été « oublié ».5 Je dis troublante parce qu'elle donne lieu à une confusion qui semble assez répandue parmi les commentateurs de l'art performatif chinois. Commentant un passage du Zhuangzi où un artiste est reconnu comme tel par un seigneur en raison de son comportement à la fois méditatif et extravagant, le critique d'art chinois Fei Dawei s'exclame, assez naïvement il me semble : "Why is that even as early as 2000 years ago, behaviour and procedure were seen as «true art » rather than the technique of painting itself? " 6 Lorsque la conscience procédurale caractéristique de l'art performatif est assimilée à une approche taoïste du corps et de l'existence, il me semble que l'on risque de commettre un malheureux contresens. ${ }^{7}$ Non pas que l'action performative ne puisse comporter une forte dose méditative (le cas de Zhang Huan est certainement le plus éloquent à cet effet, comme nous aurons l'occasion de le constater); mais parce que l'art performatif, avec son intentionnalité et son pour quoi, institue nécessairement un plan de représentation qui me semble profondément étranger au devenir-imperceptible taoïste, lequel ne cherche vraisemblablement pas à se signifier - non pas en raison d'une hypothétique insuffisance historique, mais bien en fonction d'une orientation spirituelle radicalement opposée. Le rapport taoïste au corps vécu implique un régime d'activité étranger, et à la limite, hostile au display performatif, qu'on pourrait encore décrire comme une sorte de juste-au-corps métaphysique par sa manière de faire parfaitement coïncider un plan de représentation avec les limites corporelles d'un individu. ${ }^{8}$ Et encore : si le corps est effectivement au cœur de la pratique taoïste, ce n'est certainement pas en tant que matière d'expression, comme nous le rencontrons de manière paradigmatique dans certaines des œuvres que nous discuterons plus loin.

$\mathrm{Au}$ carrefour de la conception traditionnelle chinoise du corps rituel et de l'évolution récente de l'art performatif en Chine, nous posons donc deux questions, qui débordent largement ce contexte national: quels sont les enjeux impliqués dans l'expositionperformative d'une conduite humaine? Ou dans une optique plus biopolitique : que signifie exposer/dis-play (de) l'humain? Cette réflexion sur l'art performatif en Chine s'inscrira, en dernière analyse, sur fond d'un questionnement au croisement $\mathrm{du}$ biopolitique et de l'anthropogénétique, questionnement déjà bien présent dans de nombreuses œuvres que nous allons maintenant aborder.

\section{Persistance - la performance comme action pure}


Est-il possible de s'en tenir à la contre-effectuation d'un événement, simple représentation plane de l'acteur ou du danseur, tout en se gardant de la pleine effectuation qui caractérise la victime ou le vrai patient? Toutes ces questions accusent le ridicule du penseur (...)

Gilles Deleuze, Logique du sens

\section{Évolution du contexte sociopolitique durant les années 90}

Suite aux événements de la place Tiananmen, la situation de l'art expérimental en Chine est extrêmement précaire. Les artistes expérimentaux ne peuvent exposer leurs œuvres, et les revues d'art qui s'intéressent à leur travail sont étroitement contrôlées. Deux des plus grands critiques d'art chinois encore aujourd'hui, Gao Minglu et Li Xianting, qui à l'époque étaient respectivement les éditeurs en chef des revues Meishu bao (Fine arts newspaper) et Meishu (Fine arts), furent démis de leur fonction en 1989 et 1990. Au début des années 90, l'art expérimental en Chine apparait au point mort.

À partir de 1992-93, plusieurs des artistes qui relanceront la scène de l'art performatif en Chine vivent ensemble dans un petit village en périphérie de Beijing (East Beijing village). C'est une époque de profonds changements économiques, où la Chine accélère son entrée dans l'économie de marché et adopte les standards de la société de consommation. La libéralisation économique contraste fortement avec le contrôle qui continue de prévaloir sur la sphère politique et culturelle, créant une tension identitaire énorme qui se reflète inévitablement dans la production artistique de cette période. Cette situation schizophrénique donnera ainsi lieu à des pratiques performatives qui vont progressivement s'éloigner du modèle de performance comme action publique qui prédominait vers la fin des années 80 , pour évoluer vers des pratiques qui se consacrent davantage à l'exploration du corps-soi et du corps-chair.Généralement, on peut faire l'hypothèse que la radicalisation de l'art performatif en Chine reflète la disparition progressive de la possibilité d'agir en tant que sujet symbolique dans l'espace public. L'usage du corps comme matière autonome d'expression et l'extrême cruauté qui va caractériser nombre de performances de cette période témoigneraient ainsi d'un procès général de réduction postpolitique de l'existence, lequel compromet les formes classiques de politisation du malaise socio-existentiel.

À première vue, ce cadre général d'interprétation s'applique plus particulièrement aux performances impliquant le corps-soi, c'est-à-dire celles où le corps individuel est volontairement soumis à des sévices extrêmes, et dont la valeur consiste dans la capacité du performeur à supporter la souffrance auto-infligée. Dans de tels cas, l'épreuve de la souffrance révèle un pouvoir individuel d'action inédit, qui vient défier les diktats du pouvoir. Pour Lesley Sanderson, 


\begin{abstract}
The performance is a symbolic enactment of pain and suffering. (...) The value of the artistic gesture is in the fact that the artist chooses to put himself through the pain. This signals the authenticity of his intention and the artist's need to comment on humanity, its suffering, and the lack of agency within real life situations. We interpret the willingness of the artist to take on the burden of suffering within the symbolic act of the performance as a heroic and significant act. It claims the potential of the individual's agency to stand apart from the collective and demonstrate one's accountability.9(Je souligne)
\end{abstract}

Cette description convient parfaitement aux performances de Yang Zhichao, Zhang Yuan et He Yunchang. Chacun à leur manière, ces trois artistes insistent sur la puissance individuelle d'action, ou plus précisément sur la valeur de persistance, qui est parfois soulignée par le caractère mythico-utopique et des efforts mis en scène (en particulier dans le cas de He Yunchang). Le caractère souvent héroïque des performances qui impliquent le corps-soi leur confère une valeur spécifiquement humaine qui contraste avec les installations et pratiques performatives qui utilisent le corps organique ou corps-chair comme matière autonome d'expression. ${ }^{10}$ Dans ce deuxième cas, le rapport expressiviste entre répression politique et performance ne s'applique plus qu'indirectement; et l'extrême cruauté mise en scène de relever d'une tentative passablement perverse de pousser à bout une froide logique biopolitique. D'une certaine manière, le défi de cet article consiste à marquer le seuil entre la performance entendue comme expression d'un malaise politicoexistentiel et la performance comme acting-out d'une logique biopolitique.

Vers la fin des années 90, la pression se relâche sur le monde de l'art performatif, donnant lieu à une véritable éclosion de la discipline. Au mois de mai 1999, l'artiste Ma Liuming organise le China-Japan Performance Art exchange à Beijing, qui fut le premier événement international d'art performatif jamais tenu en sol chinois. Plusieurs rencontres internationales suivront, dont l'historique Fuck Off/不合作的方式 (bu hezuo de fangshi, «approche non-coopérative»), exposition satellite faisant partie de la Biennale de Shanghai de l'an 2000. Tous les artistes que nous allons étudier figurent dans le catalogue de l'exposition; il est par contre plus difficile de déterminer s'ils étaient tous au programme de l'exposition. Comme Berghuis l'a fait remarquer, il y a un écart considérable entre les œuvres qui ont été effectivement présentées et le catalogue de l'exposition, qui s'explique par l'autocensure ou « auto-inspection » (ziwo jiankong) effectuée par les commissaires de l'exposition. ${ }^{11}$ Berghuis ajoute également quelques remarques sur la réception occidentale de l'art contemporain chinois et sa tendance à reproduire l'opposition entre avant-garde et art « officiel », qui s'appliquerait difficilement à son avis dans le contexte chinois. Si un grand nombre de commentateurs semblent, comme lui, se satisfaire de cette critique interculturelle des ratés de la réception occidentale, notre approche consistera au contraire à approfondir le dialogue en proposant des contextes d'interprétation à la mesure des enjeux soulevés par ces œuvres si controversées.

La portée politique d'une exposition comme Fuck Off, même amputée par une 
« auto-inspection », reste indéniable. Le catalogue s'ouvre sur un texte signé par les deux commissaires de l'exposition, Ai Weiwei et Feng Boyi, texte qui donne le ton en insistant sur l'irréductibilité de l'art en rapport aux cercles de pouvoir: "Fuck off emphasis the independent and critical stance that is basic to art existence (...) What will last forever is the very uncoperativeness with any system of power discourse. $\gg^{12} \mathrm{La}$ performance minimaliste de Ai Weiwei lui-même exprime l'esprit d'irrévérence générale qui caractérise l'exposition: deux photos juxtaposées nous montre l'artiste en train de faire un doigt d'honneur à la Maison blanche et à la Cité interdite respectivement. Les photos sont accompagnées d'un extrait d'un discours de Mao Zedong intitulé La tâche de la culture révolutionnaire dans cette période historique particulière :

Those comrades who are firm and determined in today's ideological struggles and those who have no fear of power and no compromise with vulgarization will be the hope of tomorrow's new culture. ${ }^{13}$

Plus habitué aux parodies grinçantes du political pop et du réalisme cynique, on sera peut-être tenté de voir dans cette citation un clin d'œil ironique au camarade Mao. Mais force pourtant est de constater un singulier parallèle entre le volontarisme révolutionnaire et des pratiques performatives extrêmes résolument « non-coopératives » et déterminées à ne pas se conformer aux diktats du pouvoir en place.

\section{Yang Zhichao: a-charné}

That sense of realness makes me believe that performers must be early marxist revolutionnaries.

Yang Zhichao

Yang Zhichao (1963) a été décrit comme un révolutionnaire qui tente de mettre en évidence certains enjeux sociaux par l'entremise de ses performances. À défaut de se démarquer par sa qualité poétique, la démarche de Yang est d'une remarquable cohérence et d'une limpidité incontestable. Le caractère extrême de ses performances exprime un implacable réalisme, une volonté absolue de littéralement s'inscrire dans le réel. De là que l'expérience de la douleur soit un élément essentiel de son œuvre, et plus encore, la force de volonté nécessaire pour la surmonter. "Seul l'expérience personnelle de la douleur me permet d'accéder à des intuitions qui ne peuvent être atteintes sur le plan de l'abstraction. La douleur est une manière d'accéder à un autre sentiment d'exister. ${ }^{14}$

Chez Yang Zhichao, cette recherche d'un rapport brut au réel par l'expérience de la souffrance ne constitue cependant pas une fin en soi. Chacune de ses performances exprime une critique sociale forte et ciblée. Dans Fort Jiayu (1999-2000), Yang se fait admettre dans un hôpital psychiatrique d'une petite ville du Gansu, sa lointaine province 
natale. Pour ce faire, il n'aura fallu que le témoignage d'un proche (en l'occurrence, sa sœur) et un paiement pour couvrir les dépenses du séjour, qui durera un mois. Dès son admission, Yang recevra un traitement aux électrochocs de plus d'une demi-heure, qui sera complété par l'administration de fortes doses de sédatifs qui conduiront finalement l'artiste à douter de sa propre condition mentale. Durant son séjour, Yang a tenu un journal qui relate son expérience et a été secrètement filmé et photographié par son beau-frère. ${ }^{15}$

La même année, et peu de temps après son arrivée à Beijing, Yang fera marquer au fer rouge son numéro d'identification personnel sur son épaule droite par Ai Weiwei (Iron, 2000). Certains ont souligné la violence du choc entre le monde rural et le monde urbain pour expliquer cette performance. En réduisant volontairement son corps à une pure surface d'inscription du symbole par excellence du dispositif gouvernemental, il me semble pour ma part que cette performance doive être lue comme une tentative radicale de réappropriation de son propre corps, une sorte de contre-effectuation primaire de la gestion biopolitique des populations à laquelle il s'est volontairement soumis lors de son séjour traumatique en asile psychiatrique.

Un mois plus tard, Yang participe à l'exposition Fuck off avec une performance qui le rend célèbre. Dans Planting grass (2000), un chirurgien-jardinier fait deux entailles de $1 \mathrm{~cm}$ $\mathrm{x} 1 \mathrm{~cm}$ dans son épaule gauche sans anesthésie, pour ensuite y planter deux gerbes d'herbe recueillies précédemment sur les berges de la rivière Suzhou, à proximité du lieu de l'opération. À première vue, ce geste semble passablement énigmatique. Dans la foulée de Iron, on pourrait encore une fois le lire comme une métaphore biopolitique, le corps de Yang apparaissant comme simple substrat pour la croissance végétale. Ce premier niveau d'interprétation ne suffit cependant pas pour rendre compte de la dimension proprement spirituelle que Yang cherche à donner à sa démarche artistique :

Pour moi, l'art a d'emblée une dimension religieuse. Je pense par exemple à ces moines bouddhistes en Chine, qui font face à la difficile tâche de rester fidèle à leurs croyances malgré la dure répression à laquelle ils sont soumis. En mettant leurs vies au service de la coexistence pacifique et en étant prêt à mourir si nécessaire, ils nous donnent le pouvoir d'être fidèle à nos propres idéaux. Ce qui se produit derrière les murs des monastères peut être porté à l'attention du public par les artistes. ${ }^{16}$

Si l'art a d'emblée une dimension spirituelle pour Yang, c'est au sens d'un croire qui s'actualise directement dans des pratiques réelles et qui donne une mesure à l'existence. Cette insistance sur le caractère spirituel de sa pratique se vérifie tout particulièrement dans Planting grass. Questionné sur le sens de cette violence auto-infligée, il répondra: «L'engourdissement spirituel est aussi une forme de violence». La métaphore de la passivité végétative prend ainsi une tournure insoupçonnée, directement adressée à l'assistance :

In a senseless atmosphere where everyone is temporarily laughing and joking, this method of seeking extremes definitely has the effect of sobering people up. You 
certainly can't let a whole nation of people go around grinning like idiots. ${ }^{17}$

L'exigence de réel qui traverse l'œuvre de Yang et la mise en jeu radicale de son existence à travers ses performances révèlent ici leur dimension conversive et parrésiaque.

Dans sa performance Earth (2004), l'offensive spirituelle menée par Yang semble se renverser pour prendre une dimension proprement cosmologique. Comme dans Hide (2002), où il s'agissait d'introduire par chirurgie (sans anesthésie toujours) un objet dans sa cuisse, l'œuvre consiste en l'implantation d'un élément étranger à l'intérieur du corps, plus précisément un morceau de terre introduit dans le haut de son estomac. Dans cette performance, Yang s'éloigne visiblement de toute critique sociale pour faire intervenir son corps comme témoin direct de l'incommensurable écart qui sépare l'homme de la nature. La tragique contingence de l'existence humaine vient ainsi symbolisée par une performance mettant à l'épreuve la différence organique propre de l'humain. À l'ère des biotechnologies et de la lecture toujours plus fine des partitions de la nature, cette performance a quelque chose de profondément primitiviste. Serait-ce une barbare tentative au scalpel de réfuter le «principe céleste » ou le jugement de Dieu en se faisant un corps sans organes? Ou serait-ce plutôt l'expression d'un volontarisme déjanté cherchant à isoler aussi radicalement que possible la forme même de l'humain? L'intérêt de Yang ne semble pas tant porter sur l'aspect technologique de la performance que sur la dimension religieuse des aspirations intimes de l'humain : "Je sais que ce désir de combler l'écart entre l'homme et l'univers ne sera jamais entièrement réalisé. (...) Même si j'accepte la douleur, mon corps rejette la terre. Tout de même, c'est simplement humain que de vouloir combler cet écart. ${ }^{18}$ À vouloir obstinément exposer les limites de sa chair, Yang, littéralement,s'a-charne; il est humain, trop humain.

\section{Le devenir-mythique de He Yunchang}

C'est une histoire que tous les Chinois connaissent, et que l'on enseigne très tôt et fort à propos à ceux qui auraient un jour décidé de s'atteler à la tâche d'étudier le mandarin. ${ }^{19}$ C'est l'histoire de «Yugong qui déplace les montagnes », 《思公移山 » (Yu gong yi shan), un vieux fou qui décida un jour de déplacer la montagne qui obstruait l'entrée de sa maison à la sueur de son front et qui, à force d'une persévérance étendue sur plusieurs générations, finit par y arriver. Les performances de He appartiennent elles aussi à cet univers fantastique. Certaines de ses performances rejouent des mythes classiques de la culture chinoise : on pensera par exemple à Tenir promesse (2003), œuvre pour laquelle il emprisonne sa main dans un bloc de ciment durant 24 heures. Le titre de l'œuvre renvoie à l'histoire d'un rendez-vous manqué sous un pont entre un garçon nommé Wei et une fille nommée Qi. Wei se présenta à l'heure prévue malgré une forte tempête. La rivière était fort agitée, Wei s'accrocha à une des colonnes du pont et continua d'attendre, pour 
finalement en mourir. On ne s'étonnera pas non plus qu'il ait lui aussi tenté de Déplacer une montagne (1999), ou de couper une rivière en deux avec un couteau et le flot de son sang (Dialogue avec l'eau, 1999), ou encore de déplacer le soleil (Soleil doré, 1999).

Le ciment revient à plusieurs reprises comme élément auquel opposer sa force de volonté. Dans Un sac de ciment (2004), c'est toute l'industrie de la construction chinoise, laquelle accapare près de $70 \%$ de la production mondiale de ciment, qui se trouve ainsi mise au défi. Attaché par les pieds à une grue, He Yunchang déplace des sacs de ciment d'un point $\mathrm{A}$ à un point $\mathrm{B}$, pendant qu'une grue l'imite en respectant le même rythme. Dans Au-delà de Tianshan (2002), He pousse un énorme bloc de ciment dans la direction d'un canon qu'il a lui-même fabriqué et qui fait exploser $1,25 \mathrm{~kg}$ de poudre à canon, donnant lieu à une explosion spectaculaire. Dans Casting (2004), il s'enferme durant 24 heures dans un bloc de ciment. Dans Ordre d'un général (2005) enfin, il trempe son corps jusqu'à la hauteur de sa poitrine dans un bloc de ciment encore liquide. La performance durera 1 heure, pendant laquelle le ciment durcira lentement, et He Yunchang s'en sortira avec d'innombrables coupures sur tout le corps. Ces coupures rappellent la manière dont Canetti décrit le mode d'action psychologique du mot d'ordre : ils se plantent comme des aiguillons dans la chair de ceux qui les reçoivent, ne laissant à ceux-ci d'autre choix que de les passer le plus vite possible à d'autres. Tout se passe comme si par cette performance, He cherchait à concrétiser l'interruption de ce processus de cimentification social, en résistant jusqu'aux limites du supportable à l'exécution de "l'ordre du général». L'immédiateté corporelle de la performance en fait une forme d'art particulièrement efficace pour court-circuiter, au moins symboliquement, la vicariété intrinsèque du pouvoir, c'est-à-dire, la fonction de représentation et de délégation essentielle à sa consolidation.

Contrairement à Yang, l'essentiel chez He ne consiste pas dans le fait de la blessure, mais plutôt dans la création d'une situation de corps-à-corps avec une puissance matérielle donnée, confrontation qui vient mettre en valeur la force de résistance d'une volonté individuelle. Cette affirmation souffre toutefois d'une notable exception : dans Test de vision (2003), He fixera son regard sur un ensemble d'ampoules de 10 ooo watts accrochés à un miroir pendant une durée d'une heure, ce qui lui causera des dommages irréparables à la vue. On peut également penser à sa performance aux chutes Niagara (2005), où, équipé d'une corde d'escalade, He brava une température de 3 degrés Celsius et s'accrocha à la paroi rocheuse. La performance fut interrompue par l'intervention des policiers, sans quoi il aurait bien pu mourir de froid.

Comme dans le cas de Yang, les performances de He exigent une grande endurance physique et une forte détermination. Le conflit instauré entre le corps et l'environnement extérieur devient une manière d'éprouver la puissance du soi. Plutôt que de rabattre l'existence humaine sur sa limite physique, les performances de He instaurent un plan de représentation à mi-chemin entre l'extrême dureté du réel et la puissance du mythe. La 
performance entrouvre une dimension mythique, d'où émergeront des forces pour affronter l'intraitable réel. "Rien ne peut nous empêcher de recourir à une imagination sans limite lorsque nous sommes confrontés à des circonstances adverses », dit-il, ajoutant que cet esprit utopique lui est inspiré par la remarquable détermination qu'il trouve chez les gens défavorisés. Les performances de He s'articulent ainsi comme des « contes pour adultes » selon sa propre expression, qui mettent en scène la puissance de la volonté individuelle dans le but de «créer de l'espace pour l'imagination ${ }^{20}$. L'importance accordée à la vertu de persistance dans la culture chinoise trouve ainsi dans l'œuvre de $\mathrm{He}$ une illustration exemplaire - à hauteur d'homme.

\section{Zhang Huan en méditation}

À l'intérieur, je suis un bouddhiste; à l'extérieur, je suis un artiste.

Zhang Huan

La démarche artistique de Zhang Huan est riche et complexe, travaillée de l'intérieur par une forte exigence spirituelle qui s'exprimera aussi bien dans l'extrême ascétisme des premières performances que dans les œuvres monumentales plus récentes qui témoignent de sa conversion au bouddhisme. Sa première performance publique est assez peu connue et s'intitule Ange (1993) : sur un grand drap blanc étendu sur le sol à l'entrée du Musée national d'art de Beijing, il lance une jarre remplie de peinture rouge sang et de fragments de poupée. Cette performance, réalisée à une époque où toute performance était interdite, conduira à la fermeture de l'exposition et l'obligera à payer une amende de 2000 Yuans. La critique politique relativement explicite contenue dans cette œuvre (référence à la politique de natalité chinoise et/ou au massacre de la jeunesse étudiante de place Tiananmen) contraste fortement avec l'évolution ultérieure de son travail, ce qui explique pourquoi cette première performance est souvent négligée.

C'est au sein de la communauté d'artistes du East Beijing Village que Zhang fera sa marque, avec une série de performances extrêmes qui ont fait forte impression. Dans 12 mètres (1994), il s'enduit de miel et de sauce à poisson et s'installe dans une toilette publique du village. Pendant près d'une heure, son corps est envahi par une nuée de mouches qui ne le quitteront que lorsqu'il se sera complètement immergé dans un étang pollué situé à proximité des toilettes. La description quasi-phénoménologique de cette performance offerte par Zhang nous plonge d'emblée dans la profondeur méditative de son travail :

I just felt that everything began to vanish from my sight. Life seemed to be leaving me far in the distance. I had no concrete thought except that my mind was completely empty. I could only feel my body, more and more flies landing and crawling over my nose, eyes, lips, ears, forehead, every part of me. I could feel them eating the liquid on 
my body. Some were stuck but did not stop eating. I could even tell that they were more interested in the fish liquid than the honey because there were more flies on the left part of my body, where that liquid was. The very concept of life was then for me the simple experience of the body. ${ }^{21}$

En regard de cette performance, Zhang rappellera également qu'elle n'a fait que refléter l'expérience ordinaire des gens qui doivent quotidiennement fréquenter ce lieu public. Cette insistance sur le caractère ordinaire de l'existence est à la source du travail de Zhang, marquant dès ses débuts une proximité avec la conception bouddhique du sacré comme banal quotidien. Mais de toute évidence, il est difficile de nier que dans ses premières performances, c'est plutôt l'élément cathartique qui prime. Dans $65 \mathrm{~kg}$ (1994), Zhang se suspend 3 mètres au-dessus du sol avec l'aide de chaînes. Il a préalablement inséré deux tubes de plastique dans ses bras, desquels s'écoule son sang, qui tombe sur une plaque de métal chauffée par un brûleur. La puanteur du sang brûlé s'imprègne dans les matelas qui couvrent les murs de la pièce, ajoutant au dégoût et à l'impression d'enfermement éprouvé par l'audience. Cet exercice masochiste en grand style s'intitule $65 \mathrm{~kg}$, en référence au poids de Zhang avant la performance. Cette référence mathématique à la pure corporalité (12 mètres était aussi signé d'une même numérale impersonnalité) nous renvoie d'autant plus certainement à l'idée de performance comme extraction de vie nue. Parlant de 12 mètres et de $65 \mathrm{~kg}$, il dira : "Dans mes performances, j'essaie de faire l'expérience du corps et de la réalité - survie. Je méprise l'aspect performatif de mes œuvres. » C'est dans la mise en survie performative que Zhang explore le rapport entre le corps et l'esprit, pour ainsi atteindre à la cruauté de l'existence :

In performance, I try to let my mind leave my body and forget the surrounding conditions. At that moment, I cannot feel any pain. Yet, the mind cannot really leave the body. Instead, it keeps going back to the body. And when the mind returns to the body, there comes an ever stronger feeling of the body's real situation. It makes you more conscious of the cruelty of the reality and makes you feel more uncomfortable. But it is not the physical pain in the physical body, but rather the spiritual uneasiness.22(Je souligne)

Le désir de cruauté de Zhang, ce désir de faire une expérience corporelle immédiate et fulgurante du réel, demeure inscrit dans une démarche spirituelle orientée par la recherche d'un effet cathartique, sur lequel il revient à de nombreuses reprises et qui compte davantage à ses yeux que l'endurance physique même :

I try to experience the relationship between the physical body and the spiritual body in particularly designated circumstances. I want to make this experience clearer and deeper in some radical situations. Not just for the sake of testing the endurance of my physical body under external pressure, but rather through this process of endurance $a$ deeper panic in the spirit might be released, though perhaps just temporarily.23(Je souligne) 
Cette violente quête cathartique définit un premier temps dans l'œuvre de Zhang. Sur le plan existentiel, elle préfigure vraisemblablement, à quelques dix années de distance, sa conversion au bouddhisme. Entre-temps, le travail de Zhang acquiert une remarquable dimension poétique. Ajouter un mètre à une montagne inconnue (1995), œuvre à laquelle plusieurs artistes du East Beijing Village ont participé mais dont il réclame la paternité, rejoue le thème de la vie nue avec une grande sobriété. L'accumulation des corps en une pyramide anonyme sur le sommet d'une colline des environs de Beijing a quelque chose de profondément émouvant. De même avec Augmenter le niveau d'eau d'un étang (1997), une autre œuvre collective qui met en scène une quarantaine d'hommes, pour la plupart des travailleurs migrants. Une grande force tranquille émane de ces singularités quelconques ex-posées sur le seuil indécidable de la vie nue. La puissance d'expression de cette œuvre repose là encore sur le fait que les corps ne valent qu'en vertu de leur simple volume dans l'espace, ainsi que sur la tension dynamique entre l'élément aquatique yin, et ces innombrables corps d'homme qui se tiennent debout, l'eau à mi-corps, face à l'objectif.

En 1998, Zhang réalise 1/2 (fig. 1), dont le titre exprime la bipartition entre corps et esprit. Zhang se couvre successivement d'une côte de porc et de caractères chinois inscrits par les spectateurs présents. Cette performance au seuil de la vie organique animale et de l'inscription culturelle préfigure la quête identitaire qui va caractériser ses œuvres à venir. Quelques mois plus tard en effet, Zhang s'installe à New York. Il y restera jusqu'en 2006, date où coïncident grosso modo sa conversion au bouddhisme et son retour en Chine, à Shanghai plus précisément. À New York, Zhang aimait à dire que son studio, c'est son cerveau; à Shanghai en revanche, il s'installe dans un immense entrepôt qui lui laisse, ainsi qu'à son équipe de production, tout le loisir de créer d'immenses sculptures souvent inspirées par la figure majestueuse de Bouddha. Deux des œuvres les plus fascinantes de Zhang ont été créées peu après son arrivée à New York et témoignent du choc culturel qu'il a subi. Dans Pèlerinage : fengshui à New York (1998), une performance présentée dans le cadre de la célèbre exposition "Inside Out » consacrée à l'art contemporain chinois, Zhang Huan accomplit un rituel de prière bouddhiste avant de s'étendre durant six longues minutes sur un lit de glace de style antique chinois, avec des chiens attachés aux quatre coins. La prière bouddhique et la référence au fengshui, cet art traditionnel de l'habitation chinois désormais si populaire en Occident, soulignent à la fois les origines culturelles de Zhang et les efforts entrepris pour s'orienter dans son nouveau milieu. Le choc des cultures est dramatiquement symbolisé par sa tentative de rester couché sur le lit de glace. La chaleur du corps de Zhang, son brûlant désir de s'établir dans une nouvelle culture suffiront-t-ils à faire fondre le lit des traditions duquel il est issu? La position est intenable. Autour, les chiens aboient. Ils représentent, selon les dires de Zhang, la diversité culturelle de New York... Gao Minglu offre une très belle analyse de ce dernier élément symbolique, qui donne une dimension insoupçonnée à cette représentation agonique du choc des cultures: 
In China, he was like a human animal, and because almost everyone had nearly identical physical features and attitudes, he was able to realize in a very profound way the differences and also the relationship between his own animal-like human body and the human spirit. But in the West humans were cultural animals. ${ }^{24}$ (Je souligne)

Le problème de l'inscription culturelle reviendra sous diverses formes dans les premières œuvres new-yorkaises de Zhang. Il aura tendance à s'exprimer dans des formes moins extrêmes sur le plan corporel, comme si, effectivement, il ne s'agissait plus tant de démontrer la puissance spirituelle de l'humain que de laisser voir la situation du corps lost in translation. Dans Arbre de famille (2000), trois calligraphes tracent un jour durant des caractères chinois sur le visage de Zhang, jusqu'à le noircir complètement. Parmi les nombreuses inscriptions, histoires traditionnelles, poèmes, noms, il demande qu'on écrive sur son front - et ce n'est certes pas un hasard - la fameuse histoire de Yukong le vieux fou qui réussit à déplacer une montagne. Histoire de persistance. Action pure. Est-ce que Zhang cherche par cette performance à faire corps avec les codes-sources de sa culture d'origine, comme le titre de la performance semble l'indiquer? Le titre d'une œuvre comme Keep Rooting (2003) par exemple, et plus généralement, l'importance croissante que prendra la tradition bouddhique dans son travail, nous donne toutes les raisons de le croire. Zhang se peint le visage : c'est un artiste. À l'intérieur...

Une autre lecture de cette œuvre reste possible, malgré la volonté explicite d'enracinement de Zhang, qui nous permettrait de traverser le plan de représentation identitaire. Une lecture anti-généraliste qui, au lieu d'insister sur le rôle fondateur du signifiant culturel comme marqueur comportemental et puissance de visagéification, mettrait plutôt l'accent sur sa contre-effectuation performative. Il y a un visage, plan d'inscription bioculturel, qui progressivement s'efface sous la prolifération de signes, lesquels s'annulent mutuellement jusqu'à ne plus signifier que la sur-face du langage. Et il y un corps, un corps désormais sans visage, un corps qui persiste et signe : il y a du langage, il y a la vie; et il y a une idéalité performative où l'individu se saisit comme événement.

Le problème est donc de savoir comment l'individu pourrait dépasser sa forme et son lien syntaxique avec un monde pour atteindre à l'universelle communication des événements (...) Il faudrait que l'individu se saisisse lui-même comme événement. (...) Chaque individu serait comme un miroir pour la condensation des singularités, chaque monde une distance dans le miroir. Tel est le sens ultime de la contre-effectuation. ${ }^{25}$ 


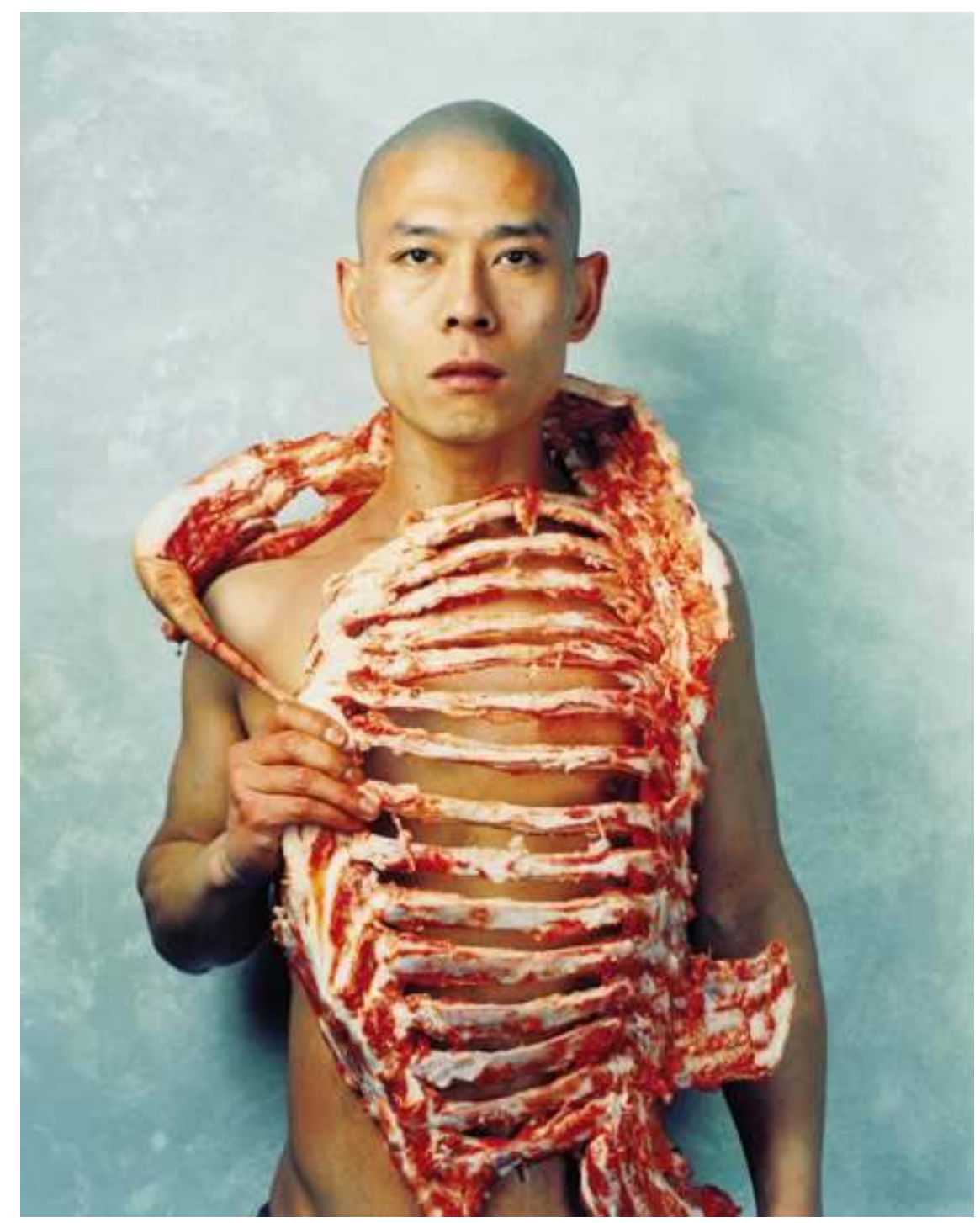

Figure 1: Zhang Huan, 1/2, performance, 1998

\section{Du corps-soi au corps-chair}

Humain : tu n'es représenté ni par ton corps ni par ton cadavre.

Peng $\mathrm{Yu}$

L'a-charnement de Yang Zhichao, le devenir-mythique de He Yunchang et les catharsis 
spirituelles de Zhang Huan constituent autant de démarches dont l'exigence première consiste en un dépassement de soi dans la performance. Le corps y est mis en jeu de manière telle à mettre en valeur la force de volonté individuelle et son potentiel héroïque de résistance. Bien sûr, le corps y est à l'honneur; mais le rapport souffrance-ascétisme qui sous-tend ces performances constitue, ultimement, une affirmation de la puissance de l'esprit humain, ou encore de la force morale propre d'un individu comme il est commun de dire.

Quelque chose de sensiblement différent est en jeu dans cet autre courant d'art extrême que nous allons maintenant aborder et qui figurait lui aussi au programme de l'exposition Fuck off. À peu près à la même époque, c'est-à-dire vers la fin des années 90, émerge une série d'œuvres qui, plutôt que de mettre à l'épreuve la forme sensible de l'humain, cherche plutôt à en questionner la définition même. Dans Proches parents (1996) par exemple, Feng Weidong s'immerge dans un aquarium en compagnie des cadavres de 32 canards, poulets et oies et de 60 livres d'intestin de porc. Le titre de l'œuvre souligne une volonté d'interroger la différence entre l'humain et l'animal qu'on retrouvera dans de nombreuses œuvres subséquentes. Le statut de la corporalité humaine est également radicalement mis en cause par l'usage de la chair humaine comme matière autonome d'expression. La performance-vidéo Arc-en-ciel (1998) de Xu Zhen apparaît comme un cas liminaire qui donne un premier aperçu de la manière dont la chair vient isolée en tant qu'élément expressif propre, marquant ainsi une distance avec les performances extrêmes impliquant le corps-soi décrites précédemment. La vidéo, d'une durée de 4 minutes, montre le dos nu d'un individu anonyme dont la couleur change graduellement à mesure qu'il se fait fouetter. Dans cette ouvre, ce n'est pas tant la capacité de supporter la douleur que la réaction physiologique de la peau battue qui se constitue en valeur d'expression.

Deux expositions tenues à Beijing un peu avantFuck offmarquent l'émergence de ce nouveau courant artistique: Post-sensibility: Alien bodies and Delusion(1999)(Hòu ganxìng: yìxíng yu wàngxiang) et Infatuation with injury(2000)(Duì shanghai de míliàn). Ces deux expositions se distinguent par la présence de nombreuses œuvres impliquant l'usage de cadavres d'humains et d'animaux, au point que plusieurs critiques parleront de l'émergence d'une «école du cadavre » ainsi que d'une fascination pour le meat art dans l'art d'avant-garde chinois. Parmi les œuvres les plus célèbres de ces expositions reproduites dans le catalogue de Fuck off, on trouve celles du couple Sun Yuan et Peng Yu. L'installation Miel(1999) de Sun Yuan (fig. 2) est composée d'un visage de vieillard émergeant d'un lit de glace, sur lequel est lové un bébé mort-né. Une grande sérénité émane de la scène, qui nous fait presqu'oublier qu'il s'agit de cadavres. Selon Sun Yuan, cette œuvre évoque " un acte d'amour » : "le bébé pose sa joue contre le front du vieillard. Il quête le lien d'amour ${ }^{26} »$. PourLes Corps connectés (2000) (fig. 3), Sun Yuan et Peng Yu effectuent une transfusion sanguine à deux bébés mort-nés. On voit le sang couler hors de la bouche des bébés et se répandre sur leur corps. Dans Huile d'humain (2000) 
(fig. 4) enfin, Peng Yu semble vouloir communiquer avec le cadavre d'un jeune enfant en lui injectant patiemment de l'huile d'humain. La sollicitude témoignée aux jeunes cadavres dans ces deux performances est profondément perturbante. Comme si le lien social, l'humanité qui nous lie à autrui était violemment exposée pour elle-même, isolée et réduite à un flot de sang et d'huile ; comme si le fait de soigner des cadavres, plutôt que de témoigner d'un souci pour le prochain, avait pour effet de profaner ce qui nous unit concrètement aux autres vivants. L'indistinction entre les vivants et les morts introduite par l'usage de cadavres humains nous amène sur un seuil où la vie humaine se voit systématiquement rabattue sur sa dimension biologique. Ces performances accomplissent l'isolation radicale du fait relationnel humain - en chinois, on pensera à $1=$, ren, la vertu d'humanité, caractère composé du radical de l'homme et du chiffre 2, suggérant qu'il n'est d'humanité qu'en relation.

Quelque chose de l'ordre du malaise issu de cette indistinction biopolitique et de l'effet de mise en suspension qu'elle engendre sur le plan existentiel me semble pouvoir être lu dans cette définition de la post-sensibilité proposée par Qiu Zhijie, co-commissaire de l'exposition du même nom : " post-sensibilité est le sentiment de doute à propos du lien de sang qui te lie à tes parents, et le sentiment que n'importe qui sur la rue est pareil à toi. » Déracinement familial, expérience d'une sorte d'anonymat urbain : la description de la post-sensibilité n'aurait-elle pas quelque chose de profondément bloomesque? $\mathrm{Ne}$ trouve-t-on pas l'expression d'une violence de cet ordre dans la performance vidéo de $\mathrm{Xu}$ Zhen brillamment intitulée But I don't need anything (1998) (而不需要什么, (er bu xuyao shenme), où un chat préalablement étranglé à mort est violemment battu sur le sol pendant 48 longues minutes?

L'usage de la chair humaine et les nombreux cas d'extrême cruauté envers les animaux dans l'art extrême chinois de ces dernières années ont souvent été lu comme une « metaphor for what flows raw, foetic and barbaric beneath the carapace of civilization. » 27 Plusieurs sont également prompts à souligner la perte de repères moraux due à la destruction du cadre de valeurs traditionnel dans la société chinois post-maoïste. Je ne crois pas qu'aucune explication moraliste ou humaniste de ce genre ne soit en mesure de rendre compte de la puissance expressive propre d'œuvres parmi les plus radicales jamais réalisées sur la scène mondiale. Il ne s'agit certainement pas d'un défaut de civilisation, ou d'un retour à la barbarie originelle qui menacerait constamment de ressurgir des tréfonds de nos âmes policées, comme si la Chine avait été, pendant quelques années, le maillon faible du système éthico-civilisationnel mondial par où se seraient échappées des forces primitives et indomptées. Au contraire, il me semble qu'il faille lire dans ce foisonnement de performances extrêmes profanant l'idée d'humanité sur fond organique pur quelque chose de très actuel et de très réfléchi - quelque chose comme un acting-out d'une logique biopolitique intrinsèquement liée à l'intégration dans la sphère capitaliste globale.$^{28} \mathrm{En}$ guise de conclusion à ce travail, nous allons essayer de compléter cette réflexion sur le 
rapport entre ce type de performance et la question du biopolitique et de la vie nue par l'entremise d'une analyse détaillée de l'œuvre de Zhu Yu.

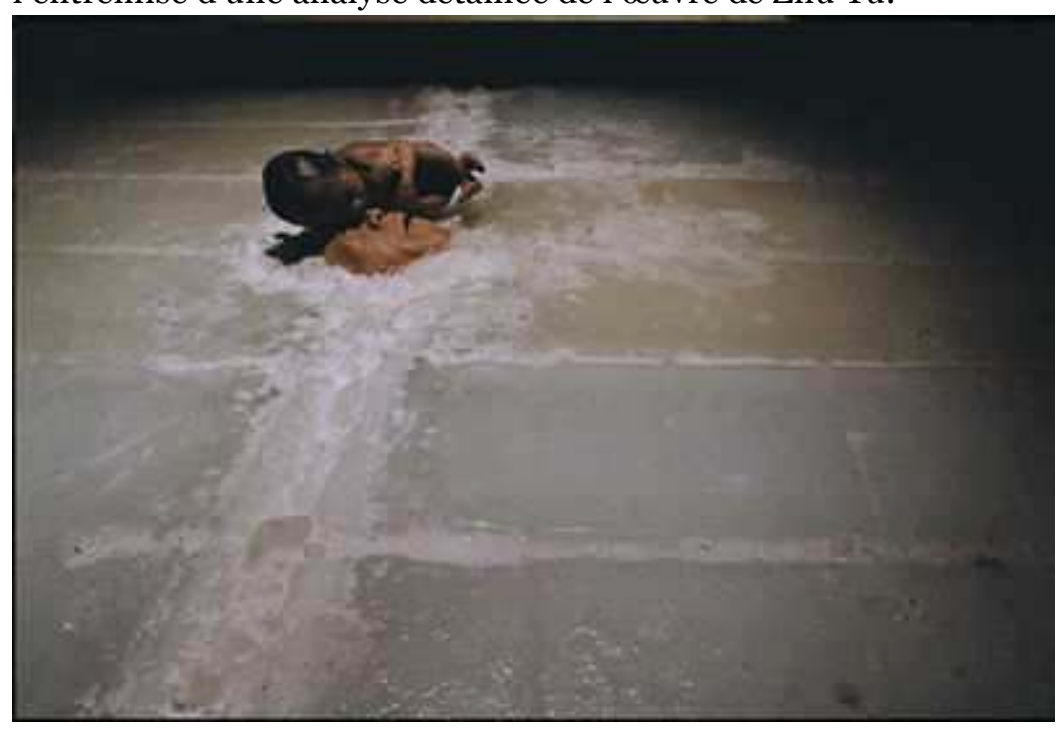

43

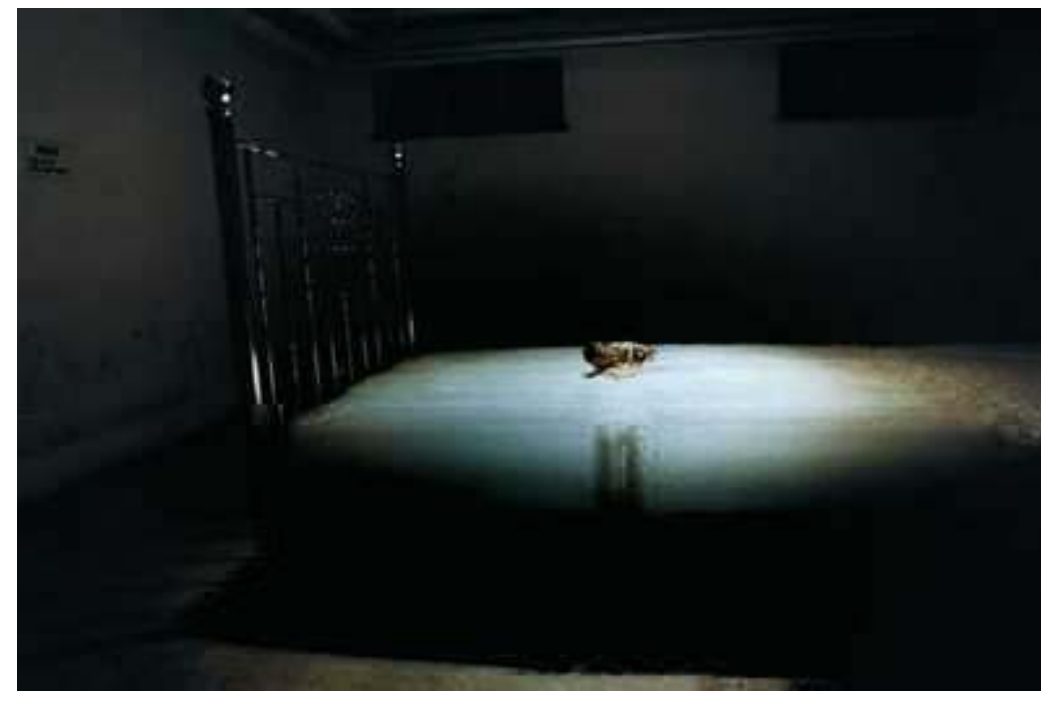

Figure 2 : Sun Yuan, Miel, installation, 1999 


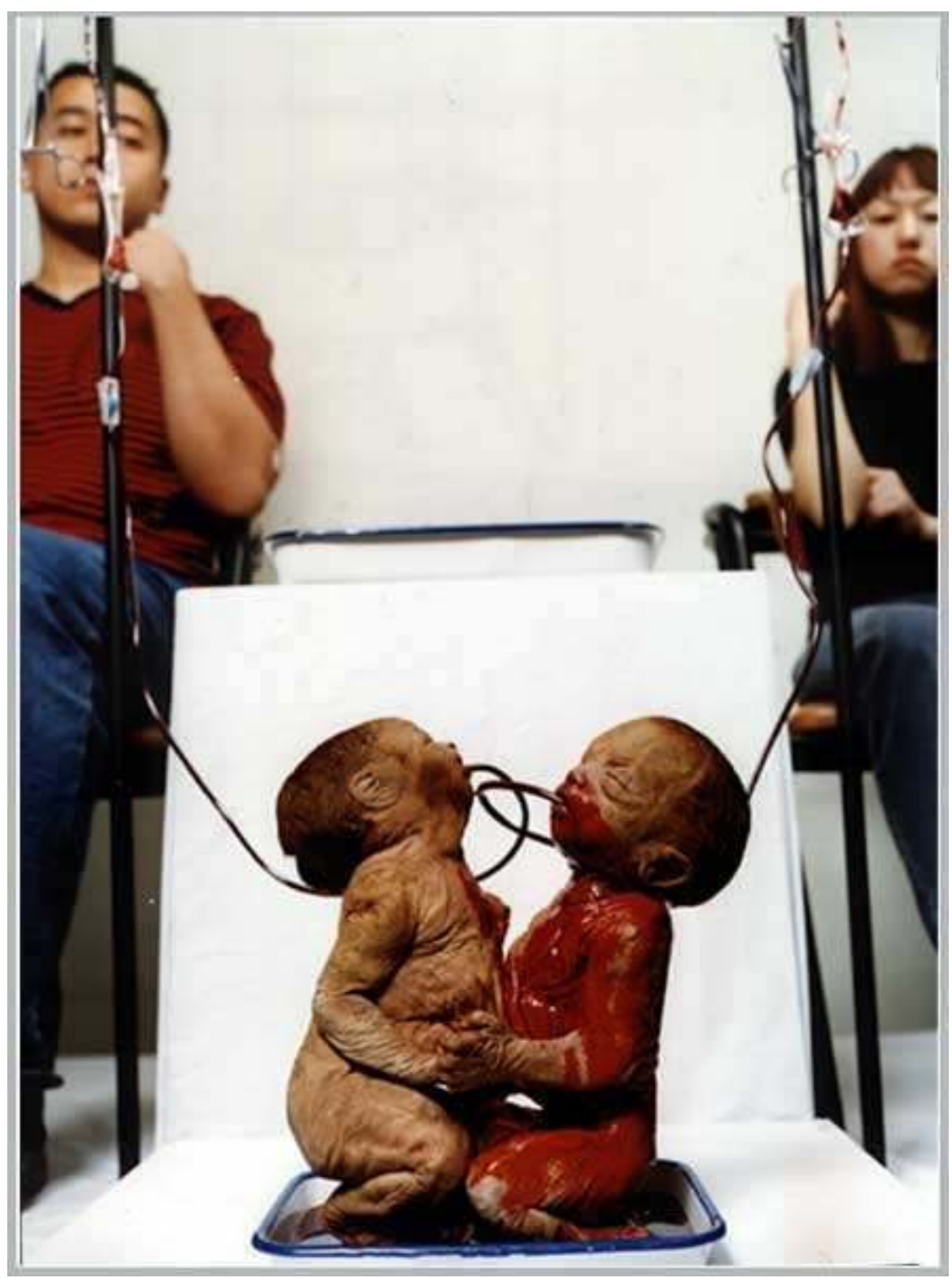

Figure 3 :Sun Yuan et Peng Yu, Corps connectés, performance, 2000 


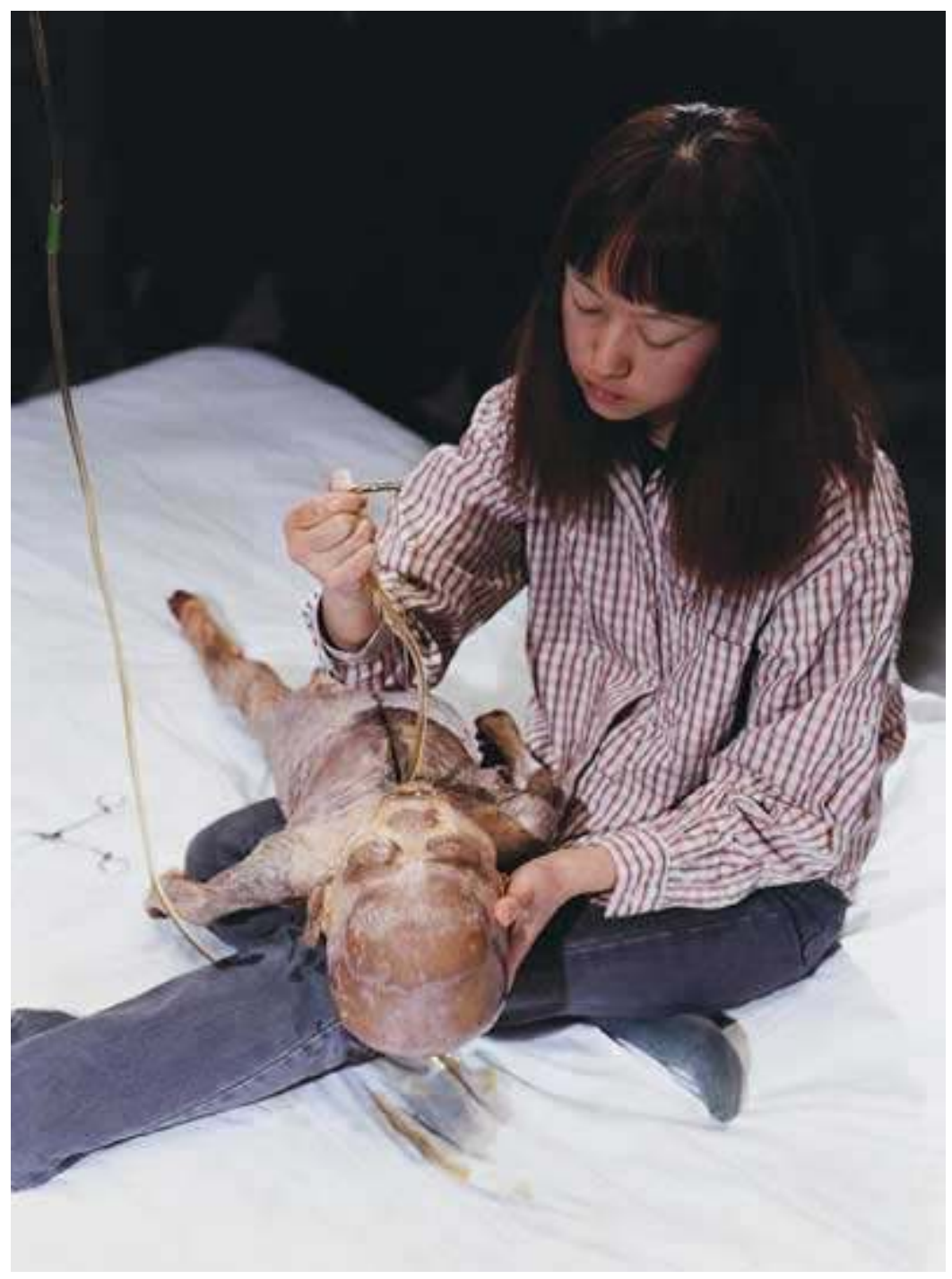

Figure 4 : Peng Yu,Huile d'humain, performance, 2000

\section{De la performance comme extraction de vie nue: le cas Zhu Yu}

Puisqu'on a pu « échanger ses fils pour les manger », on peut échanger n'importe quoi, manger n'importe qui. Lu Xun, Le journal d'un fou 
L'œuvre de Zhu Yu est sans doute l'une des plus nihilistes de toute l'histoire de l'art. En effet, rares sont les démarches artistiques qui sont allées aussi loin dans la mise à nu et la déqualification de la vie humaine. La radicalité de son ouvre réside dans une tentative systématique pour rabattre l'idéalité de l'humain sur sa corporalité propre. Cette réduction «matérialiste» classique conditionne l'ensemble de sa démarche et explique l'omniprésence de la chair dans son travail, qu'elle soit humaine ou animale, vivante ou cadavérique. Mais en fait, il serait plus exact de dire que, malgré ce que l'on serait tenté de croire, sa matière première d'expression ne consiste pas tant en de la chair proprement dite, mais en des extraits «d'essence humaine » soigneusement prélevés à même le composé fictionnel humain. Le geste performatif de $\mathrm{Zhu} \mathrm{Yu}$ - son display profanatoire est en effet empreint d'une fascination morbide pour le réel de la science, celui que l'on découpe au scalpel et qui se déchire à belles dents. Pour Zhu Yu, l'humanité n'est qu'une fiction, qu'il a un jour décidé de réduire à néant par une sorte d'acting out biopolitique primaire, qu'il a opéré en tant qu'authentique "homme sans contenu » ${ }^{29}$, maître ès extracteur de vie nue.

Si la démarche profanatrice de $\mathrm{Zhu} \mathrm{Yu} \mathrm{est} \mathrm{somme} \mathrm{toute} \mathrm{relativement} \mathrm{simple} \mathrm{et} \mathrm{univoque,}$ force est de constater que sa réception demeure, encore aujourd'hui, largement insatisfaisante, cantonnée qu'elle est à la dimension juridico-morale du scandale qu'elle a provoqué. Qu'on me pardonne donc ce rapide aparté autour du concept de vie nue, lequel me permettra, dans un deuxième temps, de situer l'œuvre de Zhu Yu dans le contexte biopolitique qui lui est propre.

\section{Qu'est-ce que la vie nue?}

Le concept de vie nue apparaît une première fois dans la célèbre "Critique de la violence » (1921) de Walter Benjamin, où celui-ci s'en prend au dogme qui affirme le caractère sacré de la vie: «fausse et basse est la proposition selon laquelle l'existence se situerait plus haut que l'existence juste, si par existence on entend seulement le simple fait de vivre [en allemand blo $\beta$ Leben, «vie nue»].»30 C'est avec Homo sacer. Le pouvoir souverain et la vie nue (1995) du philosophe italien Giorgio Agamben que le concept de vie nue devient un incontournable de la philosophie politique contemporaine. Par définition, la vie nue est abstraite: elle est le fruit d'une extraction. En ce sens, la vie nue ne désigne pas un simple état de nature, comme certains ont parfois tendance à le croire, mais doit toujours être pensée comme le résultat d'un processus par lequel se fonde un pouvoir souverain et hiérarchique. "La vie nue, dit par exemple Agamben, habite dans le corps biologique de chaque être vivant» ${ }^{11}$, ajoutant: «Elle est un produit de la machine biopolitique et non quelque chose qui lui préexiste.» ${ }^{2}$ Dans cette optique, le concept de vie nue permet d'identifier un plan où la consistance humaine est pensée à l'intersection de la souveraineté étatique et de la biologie. 
Comme chez Benjamin, le concept de vie nue chez Agamben suppose une distinction qualitative qui renvoie à l'idée d'une vie plus pleine, qui résiste à la violence souveraine: c'est l'idée d'une forme-de-vie, c'est-à-dire «une vie qui ne peut jamais être séparée de sa forme, une vie dont il n'est jamais possible d'isoler quelque chose comme une vie nue».33 Dans les traits d'union immanents à l'expression «forme-de-vie» se joue une pensée très complexe de l'image, et c'est sans doute là qu'il faut, en définitive, chercher la puissance politique inédite de la pensée d'Agamben. «L'image est le lieu, écrit Agamben, où le sujet se dépouille de sa mythique consistance psychosomatique» 34 , signifiant par là que l'ultime consistance de la vie humaine doit être cherchée sur un plan non pas biologique, mais imaginal. En somme, l'unité humaine élémentaire n'est pas le corps ou l'individu en chair et en os, mais la forme-de-vie, chargée d'images. C'est sur fond de cette pensée de l'image et de la forme-de-vie que le caractère pseudo-transgressif de l'œuvre de Zhu Yu pourra apparaître sous son jour propre, en marge des débats qu'elle a suscités concernant l'autonomie de l'art, les droits de la personne et les limites de la moralité publique. Toute la démarche de Zhu Yu peut ainsi être conçue comme une tentative de mise à l'épreuve de la consistance de «l'humain », interprétée en termes biopolitiques.

\section{Science et profanation}

Deux éléments structurent l'ensemble de l'œuvre de Zhu Yu: son goût marqué pour la profanation, et le rapport étroit qu'il entretient avec la science médicale, tant sur le plan pratique qu'épistémologique. Une des œuvres les plus intéressantes de Zhu Yu constitue sans contredit son installation intitulée Théologie de poche (1999) (袖珍神学, xiuzhen shenxué) (fig.5), présentée dans le cadre de l'exposition Post-sensibilité. Au plafond, un bras d'humain est suspendu à un crochet de métal qui rappelle ceux utilisé dans les boucheries. Le bras tient une corde qui gît en larges et multiples boucles sur le plancher, couvrant toute l'étendue de la pièce. Le fait que la corde ne se rattache à rien et qu'elle s'accumule désordonnément sur le sol suffit à créer un fort sentiment d'angoisse. La subversion de l'iconographie chrétienne de la main tendue par les cieux est efficace et sans appel : l'effet d'abandon divin est immédiat.

L'instrumentalisation de la métaphore religieuse caractérise également Joyeuses Pâques (2001) (复活节的快乐, Fuhuojie de kuaile) (fig. 6), une performance présentée dans le cadre du festival international Dakai d'art performatif de Chengdu (Sichuan). Zhu Yu planifie la mise sous anesthésie d'un porc et son opération littéralement à cœur ouvert afin d'exposer le cœur battant dans sa cavité, avant de refermer la plaie. Zhu Yu avait, semble-t-il, déjà fait un test auparavant dans une académie d'agriculture de Beijing, et le porc avait survécu à l'opération, faisant de lui un « ressuscité » selon ses propres termes, jouant ainsi sur la traduction chinoise du mystère chrétien - 复活fù huo, « retour à la 
vie »). Malheureusement, le porc utilisé lors de la performance n'aura pas la même chance. En raison d'une dose insuffisante d'anesthésiant, le porc se réveilla durant l'opération et se débattit violemment, pour finalement mourir au bout de son sang, sous le regard ahuri de l'assistance (certains artistes présents ont même tenté d'empêcher Zhu Yu de réaliser sa performance). La performance fut déclarée un échec. Notons que $\mathrm{Zhu} Y \mathrm{Yu}$ a répété à plusieurs reprises qu'il était un chrétien pratiquant. Cela expliquerait-il, du moins en partie, sa remarquable « sensibilité religieuse »?

Une logique similaire est à l'œuvre dans la performance savamment intitulée $L e$ fondement de toute épistémologie (1998) (全部知识学的基础, Quánbu zhishixué de jichu) (fig.7) Dans cette performance qui annonce le cadre conceptuel dans lequel s'inscriront celles à venir, Zhu Yu découpe et fait cuire 5 cerveaux humains, qu'il s'est procuré dans un hôpital non-identifié de Beijing. Il conditionne ensuite le jus de cerveau ainsi produit dans 80 bouteilles soigneusement décorées et identifiées à cet effet, qu'il mettra finalement en vente dans un supermarché de Shanghai ayant commandité l'événement. À la grande surprise de l'artiste, quinze bouteilles se sont vendues au prix très raisonnable de 98 yuans, c'est-à-dire l'équivalent d'à peu près $13 \$$ CAN. Il faut dire qu'en offrant du cerveau à consommer, Zhu Yu fait fond d'une croyance populaire chinoise résumée par l'expression 吃脑哺脑 (chi nao bu nao, littéralement "manger cerveau nourrir cerveau "), selon laquelle manger du cerveau contribue directement à accroître l'intelligence (idéalement celle d'un jeune étudiant sur le point de faire un examen). 35

Dans Joyeuses Pâques ainsi que dans Le fondement de toute épistémologie, la tension performative s'instaure très précisément dans la subtile mais décisive distance entre les mots et les choses. Cette idéalité, la performance tente, aussi consciemment et méthodiquement que possible, de l'annuler. Dans Manger du monde (2000) (食人, shi ren) (fig.8), performance réalisée en vue de l'exposition Fuck Off, la violence symbolique et performative n'aura pas besoin d'un titre pour se révéler. L'œuvre consiste en une série de photos de Zhu Yu en train de cuisiner et de manger ce qu'il prétend être un fotus humain. Une photo qui a circulée sur Internet en 2001 a suscité une vive controverse sur la scène internationale et a même provoqué des investigations de la part du FBI et de Scotland Yard. Zhu Yu justifie ainsi son action : "Aucune religion n'interdit le cannibalisme. Et je n'ai trouvé aucune loi interdisant de manger des humains. J'ai pris avantage de cette zone grise entre la morale et la loi pour mon travail. ${ }^{36}$ En transgressant le tabou du cannibalisme, c'est la définition même de l'humanité qui est radicalement mise en question. Par la négative, on comprend qu'il n'y a d'humanité que par définition. C'est exactement ce que Zhu Yu a à l'esprit lorsque, en réponse aux violentes réactions de dégoût de la part du public, il déclarera : «Un humain n'est-il pas simplement un assemblage d'hydrates de carbone? Et la morale n'est-elle pas simplement quelque chose que l'Homme change selon son bon vouloir en fonction de ses soi-disant besoins d'être humain au cours de son évolution? »37 Malgré son positivisme frondeur, Zhu Yu admettra avoir vomi à plusieurs 
reprises durant la « consommation » de sa performance, ce qui constitue, sans doute, une manifestation exemplaire, pour ne pas dire une attestation, de sa propre et «bien involontaire » humanité.

\section{Les humains contre-attaquent}

En 2001, une annonce est lancée par le ministère chinois de la Culture pour interdire toute activité portant atteinte aux cadavres humains au nom de l'art. Suspecté de se procurer des cadavres et des fœtus humains par voies illégales, Zhu Yu est poursuivi devant le Tribunal intermédiaire selon l'article 302 du Code Pénal chinois. Cet article stipule que toute insulte ou atteinte aux cadavres humains est interdite. En vertu de l'article 21 de la Loi chinoise sur le mariage, il est accusé d'avoir infligé la souffrance et la mort à des nouveau-nés. Il est finalement condamné pour «crime contre l'humanité» (traduction littérale du chef d'accusation).

Les profanations perpétrées par Zhu Yu ont ceci de troublant qu'elles nous exposent, aussi directement et brutalement que possible, à la question de notre humanité. Je ne souhaite pas ici rejouer le débat entre autonomie de l'art et moralité publique (et son bras légal armé), tel qu'il a eu lieu dans le contexte chinois, ni donner voix à un humanisme de bon aloi qui voudrait en faire une question de droits humains. Car, en définitive, «l'humanité» se défend-elle vraiment devant les tribunaux? Qu'est-ce donc en effet qui comparaît, dans un tel cas? Certainement pas le fin liseré spirituel ou imaginal par lequel s'active l'unité vivante et dynamique d'une forme-de-vie. Qu'on pense au célèbre Jugement de Dieu d'Antonin Artaud: la forme du jugement, que celui-ci soit moral ou proprement légal, agit comme fixum transcendant, qui nous assigne invariablement à notre étantdonné, c'est-à-dire à ce que nous sommes ou avons été - jamais à ce que l'on devient.

Il est plus intéressant à mon sens d'interroger la manière même par laquelle Zhu Yu cherche à révéler la supposée vacuité fictionnelle sur laquelle s'érige l'humain. Ce n'est peut-être qu'ainsi qu'on pourra appréhender sa monstrueuse contemporanéité. Les performances de Zhu Yu s'ordonnent comme autant d'expériences scientifiques. Elles prétendent mettre en œuvre et pousser à l'extrême le type de conscience réflexive qui fonde notre modernité et qui est privilégiée par le dis-play performatif. Dans l'expression dis-play, il faut entendre l'effort pour disjoindre ou interrompre le «jeu» de l'existence afin d'en exposer quelque ressort plus ou moins caché. Incidemment, nous faisons souvent remonter ce type de conscience-qui-expose au début de la Renaissance, au temps des premières dissections de cadavres à des fins de recherche médicale.

La démarche artistique de $\mathrm{Zhu} \mathrm{Yu}$ démontre en ce sens une cohérence proprement biopolitique. Qu'est-ce à dire? Chaque transgression qu'il opère met en jeu des représentations pseudo-scientifiques du corps, lesquelles nient la consistance imaginale des formes-de-vie. Chacune de ses performances peut ainsi se concevoir comme un 
prélèvement systématique et scientifique (dépassionné et mesuré) de vie nue, par lequel est touchée, «actionnée» si je puis dire, la limite imaginale humaine. En observant plus attentivement les photos de la performance cannibale, on remarquera que Zhu Yu n'est pas dupe de cette dimension scientifico-médicale, essentielle à son œuvre: au-dessus de lui, on trouve une image qui semble avoir été tirée d'un manuel d'anatomie, et qui expose quatre plans de dissection d'un œil. Ce «clin d'œil» autoréflexif adressé à son audience confirmera, si besoin était, son statut d'explorateur intrépide du nouveau corps biopolitique de l'humanité ${ }^{8}$ - statut à présent, on s'en doute, révoqué, pour cause d'inhumanité.

\section{Notes}

1 Gao Minglu, «Private Experiences and Public Happenings, the Performance Art of Zhang Huan”, in Pilgrimage to Santiago, 2000. www.zhanghuan.com

2 Gao Minglu, The Wall: Reshaping Contemporary Chinese Art, The Buffalo Fine Arts Academy, Buffalo, 2005, p.163.

3 Pour Gao Minglu encore, « Body art in the Chinese context is perhaps more contextualized and less conceptual than its Western counterpart. The context is affected primarily by the consciousness of an individual artists' body language as a "behaviour"; this "behaviour", moreover, is social and non-individual." The Wall: Reshaping Contemporary Chine Art, p.163-164.

4 En 2005, 41 étudiants et étudiantes de l'académie des beaux-arts de l'université de Chengdu réalisèrent une performance dans laquelle ils se dénudèrent pour former un long domino humain en forme d'arobas. Le commentaire de Shao Daosheng, chercheur à l'académie chinoise des sciences sociales, reflète de manière exemplaire le malaise de l'establishment chinois à l'égard de l'art performatif. «En fin de compte, chaque société a sa propre culture. Je me préoccupe des étudiants universitaires chinois. Si le corps nu devient un idéal esthétique pour les étudiants universitaires d'aujourd'hui, et par suite un style de vie, et bien je ne peux pour ma part que dire qu'ils sont vraiment dégradés et sans espoir. "Cité in « Should naked human body be used as an art symbol?", China Daily, 2005-07-20.

5 Tel n'était pas l'avis de Mao, mais il faut voir comment la question du corps est pour lui d'abord et avant tout une question nationale... « Our nation is wanting in strength. The military spirit has not been encouraged; the physical condition of the population deteriorates daily. This is an extremely disturbing phenomenon. The promoters of physical education have not grasped the essence of the problem, and therefore, their efforts, though prolonged, have not been effective. If this state continues, our weakness will increase further." (Une étude de l'éducation physique, 1917). Disponible en ligne (en anglais) : http://www.amoymagic.mts.cn/sportsmao.htm

6 Cité in Thomas J Berghuis, Performance art in China, Timezone 8, Hong Kong, 2006, p. 67.

7 Malgré ses remarques sur le concept de 环境 huanjing (environnement), le concept du « corps vécu » (身体, shenti) et l'idée néo-confucéenne de «faire corps avec son environnement », Berghuis reste finalement très flou sur le rapport qui s'instaure entre pratiques performatives actuelles et la conception chinoise traditionnelle du corps.

8 Sur le rapport entre individu et représentation, voir la réflexion de François Jullien dans $L e ~ n u$ impossible, Seuil, Paris, 2005. Dans une optique résolument comparatiste dont ce n'est pas le lieu ici 
de faire le procès, Jullien s'interroge sur l'absence du Nu dans la tradition picturale chinoise et pose la question suivante : « comment rendre compte de ce fait élémentaire, que la Chine n'ait jamais conçu la mimésis? » (p.64) Dans le contexte de notre travail, la réflexion est d'autant plus intéressante qu'elle établit un rapport étroit entre la production de l'humain et la question de l'exposition et de la représentation : «le moment du $\mathrm{Nu}(. .$.$) appelle un redoublement de plan, fait surgir celui d'une pure$ représentation : seul l'homme est - peut être - nu. (...) Le Nu dresse l'homme à part du monde, et l'isole (...) Aussi, même quand il connaît la tentation naturaliste, voire prétend se dissimuler en elle, le nu ne saurait masquer ce puissant travail - d'abstraction et de séparation - dont, depuis ses origines, il a fait l'objet. » (p.27) Sur fond anthropogénétique, l'abstraction du $\mathrm{Nu}$ et le display performatif semblent avoir beaucoup d'éléments en commun.

9 Lesley Sanderson, « Body Male Fatigue », Yishu, Vol.7, N.3, Mai 2008, p.83.

10 La limite entre ces deux types de performance est évidemment poreuse et difficile à tracer. En guise d'illustration, on peut opposer les opérations d'un Yang Zhichao, qui sont réalisées sans anesthésie et qui mettent en évidence la capacité d'endurance de l'artiste, et la Greffe de peau (zhi pi) (2000) d'un Zhu Yu présentée dans le cadre de l'exposition Fascination pour la blessure (对伤害的迷 恋, dui shanghai de milian) (2000), qui se fait enlever un morceau de peau pour le greffer ensuite sur un porc en ayant recours à l'anesthésie. Zhu Yu voulait ainsi illustrer « une sorte d'absurdité qui, dans la réalité, ressemble étrangement à ce morceau de peau, qui sous-entend une souffrance ou douleur cherchant la fuite en vain». Cité par Li Xianting, commissaire de l'exposition Addiction à la plaie, in «Les principales idées del'exposition Addiction à la plaie », http://person.artron.net /show_news.php?newid=16419 (visité le 23 juin 2008).

11 Voir Thomas J. Berghuis, «Considering Huanjing: Positioning Experimental Art in China ", Positions, 12:3, hiver 2004.

12 Hua Tianxue, Ai Weiwei, and Feng Boyi, eds. Fuck Off / bu hezuo de fangshi, Eastlink Gallery, Shanghai, 2000. On peut bien sûr ironiser sur le fait que l'autocensure à laquelle se sont soumis les commissaires de l'exposition est précisément une forme de coopération avec les autorités. Dans un texte publié dans le catalogue de l'exposition Le moine et le démon, tenue à Lyon en 2004, Feng Boyi relativise quelque peu la dimension politique de l'événement et insiste plutôt sur le désir d'expression individuelle de chacun des artistes. Il souligne que « la motivation de cette exposition était l'espoir que l'art chinois d'avant-garde, dans une attitude de "non-coopération" avec le discours dominant du système chinois et avec le système artistique international, se constitue et s'affirme d'une façon personnelle et indépendante, propre à l'art contemporain chinois. » Il ajoute ensuite qu'il est « difficile d'y trouver trace de prétendues résistances politique et lutte idéologique. Car (...) ce que veulent au contraire les artistes, désireux de briser la glace, c'est oublier l'idéologie ou s'en défaire désespérément pour exprimer une individualité liée à leur propre environnement. » Feng Boyi, «UnderUnderground" et autre. Sur l'art chinois d'avant-garde depuis les années 1990 », in Le moine et le démon, 5 continents, Lyon, 2004.

13 Fuck Off / Bu hezuo de fangshi.

14 Cité in Ulrike Münter, Transitional Phase: Pain, http://www.culturebase.net/artist.php?3776.

15 Pour plus de détails, voir Thomas J. Berghuis, Performance Art in China, p.182-183.

16 Cité in Ulrike Münster, Transitional Phase: Pain.

17 Cité dans Lesley Sanderson, «Male Body Fatigue », Yishu, Vol.7, N.3, Mai 2008, p.80.

18 Cité in Ulrike Münster, Transitional Phase: Pain.

19 La popularité de cette histoire ancienne dans la Chine moderne semble dépendre en partie du fait que Mao Zedong lui-même écrivit un article à son propos, intitulé « Comment Yugong déplaça les montagnes ». Dans cet article, Mao fait de Yugong une source d'inspiration pour le parti communiste. 
Pour plus de détails, voir http://www.ramou.net/fa/faMao-1945-Yugong.xml.

20 Cité par Li Xianting, «A Myth He Yunchang Writes and Directs and Challenges He Poses Against Himself. What I Have Learnt from He Yunchang's Works”, The Rock Touring around Great Britain. He Yunchang Art Works, 2007.

21 Issu d'un entretien avec Qian Zhijian, "Performing Bodies », Art Journal, été 1999. On peut trouver l'entretien complet sur le site de Zhang Huan, www.zhanghuan.com.

22 Entretien avec Qian Zhijian, «Performing Bodies », www.zhanghuan.com

23 Entretien avec Qian Zhijian, « Performing Bodies », www.zhanghuan.com

24 Gao Minglu, « Private Experiences and Public Happenings, the Performance Art of Zhang Huan », www.zhanghuan.com

25 Gilles Deleuze, Logique du sens, Éditions de Minuit, Paris, 1968, p.208-209.

26 Frédéric Bobin, « Deux enfants perdus de l'avant-garde chinoise », Le Monde, le 26 juin 200o, p. 25. Cité par He Qian, La représentation occidentale de la cruauté dans l'art contemporain chinois, mémoie de maîtrise présenté à l'UQAM, Montréal, 2008 (non-publié).

27 John Clark, Chinese Art at the End of the Millenium, p.22-23, cité par Berghuis, p. 115.

28 À une échelle plus locale si l'on peut dire, certains critiques ont insisté sur l'effet d'entraînement qui s'est créé à l'intérieur de ce petit groupe d'artistes pour expliquer cette surenchère de violence. Voir en ce sens l'excellent article de Meiling Cheng intitulé "Violent Capital: Zhu Yu on File", où elle propose une interpretation de son oeuvre dans la perspective de ce qu'elle nomme "le capital violent": "Thus, the recent trend toward extreme performance in China may indicate the allure of what I might term "violent capital," that is, the capital- the artistic reputation and the rewards that come with it gained by violent actions framed within the symbolic realm of art." The Drama Review, 49, 3 Automne 2005, New York University and the Massachusets Institute of Technology (MIT).

29 Voir l'essai d'Agamben sur le rapport entre esthétique et nihilisme initulé L'uomo senza contenuto, Quodlibet, Macerata, 1994.

30 Walter Benjamin, « Critique de la violence », in Euvres I, Gallimard, Paris, 2000, p.240.

31 Giorgio Agamben, Homo sacer, Seuil, Paris, 1995, p.151.

32 Giorgio Agamben, Stato di eccezione, Bollati Boringhieri, Torino, 2003, p.112.

33 Giorgio Agamben, Moyens sans fins, Payot \& Rivages, Paris, 2002 (1995), p.13-14.

34 Giorgio Agamben, «Warburg o la scienza senza nome », in La potenza del pensiero, Neri Pozza, Vicenza, 2005. p.146.

35 Voir l'analyse qu'en fait Meiling Cheng dans «The Violent Capital : Zhu Yu on File ». Selon les dires de Zhu Yu, l'hôpital qui lui a fourni les cerveaux est ensuite devenu le principal fournisseur de l'école du cadavre. Dans une perspective qui demeure largement confinée à la question de la représentation identitaire, voir également la discussion de Larissa Heinrich sur le thème de la traite d'organe dans la littérature et les arts en Chine : « Souvenirs of the Organ Trade. The Diasporic Body in Contemporary Chinese Literature and Art », in Fran Martin et Larissa Heinrich (ed.), Embodied Modernities, Hawaii University Press, Honolulu, 2006, p.126-145.

36 "Baby-eating art show sparks upset." (January 3, 2003). BBC News.

37 Fuck Off / Bu hezuo de fangshi.

38 Voir Rojas, Carlos, "Cannibalism and the Chinese Body Politic: Hermeneutics and Violence in Cross-Cultural Perception”, in Post Modern Culture, 2002, qui est peut-être le premier à avoir analysé 
le rôle du tableau de l’œil disséqué dans l'économie de cette performance.

\title{
Pour citer cet article
}

Référence électronique

Erik Bordeleau, "Une constance à la chinoise : Considérations sur l'art performatif extrême chinois ", Transtext(e)s Transcultures 跨文本跨文化 [En ligne], 5 | 2009, document 3, mis en ligne le 03 avril 2010, Consulté le 08 avril 2010. URL : http://transtexts.revues.org/index269.html

\begin{abstract}
Auteur
Erik Bordeleau

Erik Bordeleau est chercheur postdoctoral en histoire de l'art et communication à la McGill University. II a récemment complété un doctorat en littérature comparée à l'université de Montréal sur le rapport entre anonymat et politique dans le cinéma et l'art contemporain chinois, sous la direction de Tonglin Lu et Brian Massumi. II collabore à diverses revues, dont Espai en blanc (Barcelone), Yishu : Journal of Contemporary Chinese Art (Vancouver), Chimères (Paris), Altérités, Inflexions, Hors-champ et OVNI (Montréal).
\end{abstract}

\section{Droits d'auteur}

(C) Tous droits réservés 